\title{
Percepção de Marca e Consumo Conspícuo: análise do EFEITO MODERADOR NA CAMPANHA PROMOCIONAL
}

\author{
Perception of Brand and Consumption Conspicuous: analysis of \\ effect promotional campaign moderator
}

\begin{abstract}
Fernando de Oliveira Santini
Doutor em Administração. Professor e Pesquisador da Universidade do Vale do Rio do Sinos - Unisinos. Escola Superior de Propaganda e Marketing (ESPM). Faculdades Senac. Porto Algre, RS. Brasil. E-mail: santiniconsultores@gmail.com
\end{abstract}

\section{Wagner Junior Ladeira}

Doutor em Administração. Professor e Pesquisador da Universidade do Vale do Rio do Sinos - Unisinos. Escola Superior de Propaganda e Marketing (ESPM). Faculdades Senac. São Leopoldo, RS. Brasil. E-mail: wjladeira@gmail.com

\section{Claudio Hoffmann Sampaio}

Doutor em Administração. Professor Pesquisador da Pontifícia Universidade Católica do Rio Grande do Sul, PUC/RS. Porto Alegre, RS. Brasil.E-mail: csampaio@pucrs.br

\section{Clecio Falcão Araujo}

Doutorando em Administração. Professor Pesquisador da Pontifícia Universidade Católica do Rio Grande do Sul, PUC/RS. Porto Alegre, RS. Brasil. E-mail: clecioa@bol.com.br

\section{Resumo}

As campanhas promocionais podem ser divididas em monetárias e não monetárias. Estas podem agir como moderadoras na intenção de compra. Nesse contexto, o presente estudo tem o objetivo de analisar os efeitos moderadores do tipo de promoção nas relações entre consumo conspícuo e percepção de marca na intenção de compra de um produto em oferta. Para isso, foi realizada uma pesquisa experimental de laboratório com 303 pessoas, simulando a intenção de compras em netbook que estavam em oferta. Os resultados apresentam uma discussão da promoção monetária $e$ não monetária agindo como moderadores na percepção da marca e na predisposição ao consumo conspícuo. Com relação à percepção da marca, não se recomenda a utilização, em demasia, de campanhas monetárias, como é o exemplo de desconto, em marcas tradicionalmente reconhecidas, pois isso poderá acarretar, em longo prazo, depreciação da imagem. Além disso, as campanhas não monetárias, possivelmente, não trarão esses efeitos negativos, considerando que essas ações agregarão valor original ao produto, sem manipular o preço tradicional.

Palavras-chave: Promoção de Vendas Monetária. Promoção de Vendas não Monetária. Marca. Consumo Conspícuo.

\section{Abstract}

The promotional campaign can be divided into monetary and non-monetary. These can act as moderators in purchase intent. In this context, this study aims to analyze the moderating effects of the type of promotion in the relations between conspicuous consumption and brand perception in the intention to purchase a product on offer. For this, we applied a laboratory experimental survey of three hundred and three respondents, with simulating the intention of netbook purchases that were on offer. The results present a discussion of monetary and non- monetary promotion acting as moderators in brand awareness and predisposition to conspicuous consumption. With regard to brand awareness, it is not recommended to use too much of monetary campaigns, as is the case off, in traditionally recognized brands, because it may cause in the long run, a depreciation of the image. On the other hand, the non-monetary campaigns possibly not bring these negative effects, whereas these actions will add unique value to the product, without manipulating the traditional price.

Keywords: Sales Promotion Monetary. Non-Monetary Sales Promotion. Brand. Conspicuous Consumption. 


\section{INTRODUÇÃO}

Os estudos relativos à perfomance das campanhas promocionais iniciaram em periódicos seminais e podem ser vistos atualmente nos debates do mainstream de marketing (LOWE; BARNES, 2012; DIELS et al., 2013; BUIL et al., 2013; LARAN; TSOIRUS, 2013; HORVÁTH; FOK, 2013), inclusive nos principais periódicos do Brasil (SANTINI et al., 2015a. SANTINI et al., 2015b; SANTINI et al., 2015c), . Apesar de muitas discussões neste período, a escolha das melhores práticas promocionais e seus principais impactos ainda são grandes incógnitas para pesquisadores e gestores do marketing (FREO, 2004; ALVAREZ; CASILLES, 2005).

Uma dessas incógnitas, em se tratando de campanhas promocionais, está nos estudos de promoção e remete ao fato de as campanhas publicitárias serem benéficas ou maléficas a percepção de marca, evidenciando assim dois eixos de estudos divergentes. Existem estudos (CHANDON et al., 2000; KNOW; UNCLES, 2005; ALVAREZ; CASILLES, 2005; SHUKLA, 2008; SANTINI et al., 2015) que indicam que dependendo do tipo de promoção, estas podem se prejudiciais a percepção hedônica do produto $e$ influenciar diretamente na percepção da marca. No entanto, paradoxalmente, existem estudos (BAWA; SHOEMAKER, 1987; ESTABA-BRAVO et al., 2009; SANTINI, 2013) que detectam o efeito positivo da promoção de vendas sobre a marca de um produto, beneficiando assim percepção hedônica do produto. Além do efeito na percepção da marca, este último argumento sustenta que a promoção pode gerar o consumo conspícuo, pois este é sustentando na exibição destacada e extravagante do produto (PATSIAOURAS; FITCHETT, 2012).

Com base nesses dois eixos, pode-se constatar que os achados dos estudos sobre os efeitos das campanhas promocionais não têm um trajeto linear, pressupondo assim, a existência de efeitos moderadores (sejam estes promoções monetárias ou não monetárias) que venham a influenciar o comportamento no ato do consumo. Essa argumentação, corrobora com alguns estudos (LOW; MOHR, 2000; FREO, 2004; ALVAREZ; CASILLES, 2005) que indicam a necessidade de novos estudos que avaliem os efeitos moderadores que possam ser fatores minimizadores ou potencializadores do impacto das promoções de vendas a curto e longo-prazo.
Desse modo, partindo do pressuposto que as campanhas monetárias e não monetárias podem agir como efeitos moderadores na intenção de compra, o presente estudo traz como objetivo analisar os efeitos moderadores do tipo de promoção na percepção conspícua e de marca. Estes efeitos serão analisados na intenção de compra de um produto em oferta e, para isto, aplicou-se um experimento, unificando as bases teóricas: (i) Promoção monetária versus promoção não monetária; (ii) percepção da marca; e (iii) consumo conspícuo.

\section{Levantamento Teórico e Hipóteses do Estudo}

Com a intenção de fundamentar as hipóteses deste estudo e assim alcançar o objetivo central deste artigo o levantamento teórico em um primeiro momento estudará a relação entre promoção monetária e não monetária. Logo em seguida, a promoção de vendas será relacionada com a intenção de compra, tendo a percepção da marca como agente de análise. Por fim, a promoção de vendas será associada ao consumo conspícuo.

\subsection{Promoção Monetária versus Promoção não Monetária}

As promoções de vendas são distribuídas em dois grupos: promoção voltadas para preço/monetária ou promoção não voltada para preço/não monetária. As promoções voltadas para preços são referenciadas como melhor alternativa na busca de venda em curto prazo, já que esta variável é fundamental na decisão de escolha de um consumidor (WINER, 1985; LATTIN; BUCKLIN, 1989; ALVAREZ; CASIELLES, 2005). Blattberg e Neslin (1990) assumem que esse tipo de promoção vai ao encontro da motivação de economia dos consumidores. Alguns estudos apontam que os consumidores sempre dão respostas a campanhas de desconto (DAVIS; INMAN; MCALISTER, 1992; NIJS et al., 2001; TAYLOR; NESLIN, 2005). Promoções voltadas para preços são baseadas em incentivos transacionais, que proporcionam recompensas imediatas e benefícios utilitários (CHANDON et al., 2000; KWOK; UNCLES, 2005). Para Tan e Chua (2004), essa técnica é avaliada pelos consumidores como uma redução de perda. 
No que tange às promoções não voltadas ao preço, os benefícios nem sempre são relacionados ao aumento de vendas em curto prazo (NBUDISI; MOI, 2005), porém eles podem estar relacionados a entretenimento e ações voltadas em longo prazo como reforço de marca (CHANDON; WANSINK; LAURENT, 2000; KWOK; UNCLES, 2005; BOSCHETTI, 2012). Algumas das ações de promoções não voltadas a preços têm como primeiro objetivo a venda, enquanto outras têm como interesse a promoção da comunicação da marca (LEE, 2002).

Nesse contexto, fica observado que, a partir dos dois tipos de promoções de vendas, os efeitos no comportamento do consumidor são diferentes. Alguns autores, por exemplo, Blatterger e Wisniewki (1989) e Alvarez e Casielles (2005), argumentam que as promoções de vendas voltadas a preços têm a preferência dos consumidores e são as mais utilizadas no mercado, sendo também as mais estudadas academicamente (GILBERT; JACKARIA, 2002; LIAO, 2006).

Paradoxalmente, existe uma corrente na qual se argumenta que as campanhas monetárias são maléficas às marcas/produtos, já que tornam os clientes extremamente sensíveis aos preços (LATTIN; BUCKLIN, 1989; EHERENBERG et al., 1994; GEDENK; NESLIN, 1999; PAUWELS et al., 2004). Em relação às promoções não voltadas para preço, autores observam um efeito mais positivo, em longo prazo, para marca/produto ofertado (BAWA; SHOEMAKER, 1987; ESTEBAN-BRAVO et al., 2009).

Lee (2002), por meio de uma survey realizada com gerentes de marcas de diversos produtos, constatou-se que as promoções de vendas são mais eficientes que propagandas para alcançar os objetivos das empresas. Verificou, também, que as campanhas de vendas focadas em retorno financeiro (promoção voltadas ao preço) são realizadas com mais frequência do que promoções não voltadas ao preço, e, ainda, que as promoções de caráter financeiro têm a preferência dos entrevistados em relação às ações de longo prazo (promoções não voltadas para preço).

Campbell e Diamond (1990), numa abordagem semelhante, constataram que as promoções não monetárias têm o objetivo de dar valor adicional a algum produto (prêmios/bônus/concursos), enquanto as monetárias têm como foco reduzir os preços (abatimento/desconto). Os autores relatam, ainda, que as promoções monetárias são facilmente integradas ao preço, enquanto as não monetárias exigem um esforço dos consumidores para que estes realizem uma integração com o preço, pois elas são consideradas separadas do valor do bem. Verificando a influência da promoção de vendas na decisão de compra dos consumidores, Gilbert e Jackaria (2002) detectaram que as promoções de descontos (monetária) influenciaram a decisão de compra com mais força, se for comparada, a promoções não monetárias. Reforçando ainda mais esta tese, pesquisas de cunho experimental realizadas por Santini et al. (2015a; 2015b) constataram que as ações de desconto (promoção monetária) são mais efetivas que campanhas de prêmios (promoção não monetária) para influenciar a intenção de compra do consumidor. Em uma das pesquisas, a média de intenção de compra, numa escala de 5 , foi de 3,61 para a campanha monetária contra 3,15 para a ação não monetária ( $p<0.001)$. Em outra coleta, a média de intenção de compra de um produto ofertado por campanha monetária foi de 3,42 contra 3,06 do produto ofertado com uma promoção não monetária ( $p<0.001$ ). Diante do exposto, surgem as primeiras hipóteses a serem testadas:

H1 - A promoção de vendas influencia positivamente a intenção de compra do consumidor, sendo que:

H1a - A promoção de vendas monetária (não monetária) influencia com mais (menos) força a intenção de compra do consumidor.

\subsection{Promoção de Vendas e Percepção da Marca}

A influência positiva que a marca exerce na intenção de compra do consumidor é consolidada a partir de décadas de pesquisas na qual se constatam efeitos positivos para simplificação de escolha (LI, 2004), avaliação de qualidade (FORSYTHE, 1991), percepção de valor (KELLER, 1993) e diminuição do sentimento associado ao risco percebido (BLACKWELL et al., 2005). No campo da promoção de vendas, o entendimento não está consolidado, uma vez que estudos constatam relação positivas - por exemplo, Cotton e Babb (1978) e Boschetti (2012), outros, vinculações neutras - por exemplo, Neslin e Shoemaker (1989), assim como se 
observam resultados com ligações negativas (LATTIN; BUCKLIN, 1989).

Aprofundando mais o entendimento entre as promoções de vendas e sua relação com a marca, nota-se que os estudos dos quais se argumentam relações negativas, direcionam esta vinculação para campanhas de caráter monetária, haja vista que as justificativas têm como base, o fato de que os clientes se tornam essencialmente sensíveis aos preços (LATTIN; BUCKLIN, 1989; GEDENK; NESLIN, 1999; PAUWELS et al. 2004). Em contrapartida, no estudo de Davis et al. (1992), que realizou cinco testes em produtos promovidos com promoções durante o período de três meses, não se encontrou nenhum decréscimo de percepção de valor nas marcas.

Por outro lado, Cotton e Babb (1978) e Rothschild e Gaidis (1981) acreditam que se os consumidores ficaram satisfeitos com determinada marca ou produto adquirido durante uma promoção, a probabilidade de se criar atitudes favoráveis ao longo do tempo será grande. Lattin e Bucklin (1989) sugeriram que as compras efetivadas durante uma promoção têm um efeito positivo em comportamentos futuros, podendo esta ser uma consequência do reforço da preferência para a marca comprada.

Para esta pesquisa, assume-se que os efeitos venham a ratificar a última suposição, amparados nas pesquisas que pontuam estas ações como reforço de compra (DELVECCHIO et al., 2006). Adicionada a essa proposição, trabalhos, por exemplo, o desenvolvido por Rajagopal (2008), demonstra relações positivas entre a promoção de vendas e relações de longo prazo, como é o caso da concepção de uma marca. Tal constatação ganha força a partir do momento em que Gibert e Jackaria (2002) e Omatoyo (2011) demonstram correlações positivas entre as técnicas promocionais e o referido construto. Solidificando ainda mais as proposições, a pesquisa de Erden e Sun (2002) detectam que o uso contínuo de promoção de vendas reforçam percepções favoráveis a uma marca.

Fazendo a associação da marca e os tipos de promoção de vendas, acredita-se que a relação seja mais forte para as ações de caráter não monetário, uma vez que nas promoções monetárias, como é o exemplo das campanhas de descontos, é possível sugerir menor percepção de qualidade (GARRETSON; CLOW, 1999), fato este, podendo ser motivado pela sensibilidade de preço criada no cliente (LATTIN; BUCKLIN, 1989). Ao contrário, a promoção de vendas não monetária, por agregar valor original ao produto, sem desconto, deverá se relacionar com mais força com a marca, fato este já detectado em pesquisas realizadas, por exemplo, por Dark e Chung (2005). Reforçando ainda mais a proposição, Boschetti (2002) realizou experimento no contexto brasileiro e detectou que a atratividade de prêmios, distribuída em uma campanha não monetária, é uma variável significativa para influenciar positivamente a escolha de uma marca no segmento financeiro. Dessa forma, sugere-se a hipótese a seguir:

H2 - A promoção de vendas influencia positivamente a percepção de marca do produto, sendo que:

H2a - A promoção de vendas não monetária (monetária) influencia com mais (menos) força a percepção de marca do produto ofertado em promoção.

\subsection{Promoção de vendas e consumo conspícuo}

A próxima suposição é que a promoção de vendas poderá evocar um sentimento conspícuo. Num primeiro momento, o comportamento de consumo era avaliado como algo racional dentro de uma perspectiva utilitária (FISHER; ARNOLD, 1990; KANG; PARK-POAPS, 2009). Posteriormente, as pesquisas sobre motivação de compra abandonaram a noção de que a compra é apenas uma atividade da cognição e passaram a considerar os valores hedônicos que direcionam o comportamento de compra (HIRSCHMAN; HOLBROOK, 1982; HALVENA; HOLBROOK, 1986; HOFFMAN; NOVAK, 1996) e, neste caso, associado a evocação conspícua (CHUNG; FISCHER, 2001; SHUKLA, 2008; WINKELMANN, 2011).

O consumo conspícuo tem sido definido como uma ação de comprar uma variedade de produtos ou serviços, normalmente, de valores caros e não necessários, para chamar a atenção de outros (SHUKLA, 2008). Diante dessa definição fica bem claro que esta sensação é desencadeada a partir da comparação com outras pessoas (WINKELMANN, 2011). Nessa linha, os estudos evidenciam que este sentimento está vinculado à percepção hedonista (CHUNG; FISCHER, 2001; SHUKLA, 2008; WINKELMANN, 2011). 
Estudos na área de promoção de vendas, por exemplo, os realizados por Chandon e colegas (2000), Kwok e Uncles (2005) e, recentemente no Brasil, por Santini et al. (2015), vinculam os benefícios utilitários e hedônicos, este último associado ao consumo conspícuo, aos tipos de promoções - monetária e não monetária. Chandon et al. (2000), Kwok e Uncles (2005) demonstraram que promoções monetárias são mais efetivas para produtos utilitários, enquanto as não monetárias são mais eficientes para produtos hedônicos. Para os autores, as promoções de vendas monetárias e não monetárias estimulam o consumo por meio de três distintos níveis de benefícios para cada tipo de motivação: economia, maior qualidade de produto e aprimoramento de compra conveniente, no caso dos utilitários; e, oportunidade para o valor expressado, entretenimento e exploração, no caso dos hedônicos. No contexto dessa definição, os benefícios utilitários ajudam consumidores a amplificarem a aquisição utilitária de compra e aumentam a eficiência da experiência de compra, enquanto os hedônicos são recompensadores e relacionados a experiências emocionais, autoestima e prazer, característica esta, associada ao consumo conspícuo (SCOTT et al., 2013).

$\mathrm{Na}$ pesquisa experimental de Chandon et al. (2000), verificou-se que o desconto de preço (promoção monetária) foi mais efetivo do que a promoção de concurso de prêmios (promoção não monetária) para influenciar na troca da marca de um detergente para roupas (produto utilitário), enquanto promoção de concurso de prêmios alcançou melhores resultados do que descontos para influenciar a troca da marca de chocolate (produto hedônico). Nesse sentido, os autores constatam que as promoções de vendas tendem a ter maior impacto quando promovem qualidades compatíveis com os benefícios exigidos dos produtos. Observou-se, também, que as promoções monetárias foram preliminarmente observadas na sua utilidade (economia e conveniência) e benefícios de valor expressado, enquanto as promoções não monetárias foram avaliadas nos seus benefícios hedônicos (entretenimento e exploração).

No experimento realizado por Santini et al. (2015), ficou demonstrada que as campanhas de distribuição de prêmios (promoção não monetárias) foram mais efetivas para evocar a percepção hedônica de um produto enquanto as ações de desconto (promoção monetária) foram mais influentes na evocação utilitária. Dessa forma, é possivel supor que:

H3 - A promoção de vendas influencia positivamente a evocação do consumo conspícuo, sendo que:

H3a - A promoção de vendas não monetária (monetária) influencia com mais (menos) força a evocação do consumo conspícuo perante o produto ofertado em promoção.

\section{Procedimentos Metodológicos}

O presente estudo se caracterizou pela aplicação de um experimento, precedida de uma etapa exploratória e descritiva, cujo objetivo foi consolidar os cenários utilizados na etapa causal. Este tipo de pesquisa é recomendada para testar relações de causa e efeito. A etapa exploratória teve como objetivo identificar alternativas de produtos, descontos e prêmios que estavam alinhados com a realidade do público participante. Esses produtos, descontos e prêmios foram, posteriormente, validados por da etapa descritiva e, em seguida, foram manipulados na etapa experimental.

$\mathrm{Na}$ etapa exploratória, a coleta se deu a partir de dados secundários - site de compras coletivas Groupon, de onde foram analisados os produtos e descontos ofertados durante o período de 15 dias. Avaliou-se no mesmo período, as promoções de prêmios autorizadas pela Caixa Econômica Federal - Gerência Nacional de Promoções Comerciais (departamento nacional responsável pela regulamentação e fiscalização destas ações), com objetivo de verificar o tipo de prêmio mais ofertado nos concursos realizados no mercado.

Uma vez realizada a identificação dos produtos, descontos e prêmios obtidos na etapa exploratória, chegou-se à etapa descritiva que culminou na consolidação do produto mais familiar e realista à vista dos participantes, uma vez que esta iniciativa é essencial para validade interna e externa de um experimento de laboratório (WILSON et al., 2010).

Para a aplicação da etapa descritiva, ficaram estabelecidos seis diferentes produtos, aplicados individualmente através de instrumentos de pesquisa que possuíam escalas de: (i) interesse sobre o produto, de Bruner e Hensel (1998); (ii) percepção de marca, de Machleit e Wilson (1988) e (iii) consumo conspícuo de- 
rivada dos estudos de Chaudhuri et al. (2011) e Shukla (2008). Os questionários foram aplicados junto a 182 estudantes do curso de administração de empresas de uma Instituição de Ensino Superior localizada no sul do Brasil.

Deve-se ressaltar que esta pesquisa se propõe a avaliar um único produto sob a perspectiva dos sentimentos associados a ele por consumidores entrevistados e, por esta razão, a intenção não é fazer as manipulações dos conceitos pela perspectiva do produto e sim do consumidor pesquisado. Nesse sentido, considerou-se não escolher produtos que suscitassem um comportamento extremado, pois tal fato poderia interferir nos resultados da pesquisa. Nesse sentido, o produto utilizado na etapa experimental partiu da constatação do comportamento menos relacionado aos construtos utilizados (intenção de compra, percepção de marca e consumo conspícuo), obtidos por meio da análise de média, além da reflexão sobre a variação das respostas dos participantes a cada comportamento, o que foi analisado a partir da técnica de desvio-padrão. Pelo resultado apresentado nesta etapa, identificou-se o produto "netbook" como o bem que teve mais variações de comportamentos.

Identificado o produto a ser utilizado, avaliou-se o desconto médio que o referido site oferece para este produto. Esta informação foi obtida na etapa exploratória, conforme os detalhamentos já expostos. Fazendo esta avaliação, chegou-se ao percentual de $37 \%$ de desconto, que foi o utilizado no cenário do experimento. Com o mesmo objetivo e, conforme já destacado, avaliou-se as campanhas de concurso de prêmios aprovadas pela Caixa Econômica Federal em que foi possível visualizar o predomínio de sorteio de automóveis $0 \mathrm{~km}$. Dessa forma, utilizou-se este bem para manipulação dos cenários apresentados sob a forma de promoção não monetária.

O questionário final utilizado nesta pesquisa buscou obter as seguintes opiniões: (i) intenção de compra do produto (sem incidência da promoção de vendas e com incidência da promoção de vendas) obtida a partir da escala de Bruner e Hensel (1998); percepção da marca fictícia do produto ofertado Machleit e Wilson (1988) e característica conspícua do consumidor, evocada pelo produto em promoção (CHAUDHURI et al., 2011). As variáveis que compuseram as citadas escalas utilizadas no trabalho são apresentadas no Quadro 01.

\begin{tabular}{|c|c|}
\hline \multicolumn{2}{|r|}{$\begin{array}{l}\text { CONSTRUTO: INTENÇÃo de COMPRA (MiNHA INTENÇÃo dE COM- } \\
\text { PRAR o NETBOOK COM A PROMOÇão DE XXXXXX É ?) }\end{array}$} \\
\hline 01 & Improvável x Provável. \\
\hline 02 & Inexistente x Existente \\
\hline 03 & Não Plausível x Plausível \\
\hline 04 & Impossível x Possível \\
\hline 05 & Incerta x Certa \\
\hline \multicolumn{2}{|r|}{ Construto: PercepÇão da marca (A marca Alpha é?) } \\
\hline 01 & De pouca qualidade $\mathrm{x}$ De muita qualidade \\
\hline 02 & De preço baixo x De preço alto \\
\hline 03 & Não dá prestígio x Dá prestígio \\
\hline 04 & Nada original x Muito Original \\
\hline 05 & Ultrapassada x Moderna \\
\hline \multicolumn{2}{|r|}{ Construto: Característica conspícua } \\
\hline 01 & $\begin{array}{l}\text { Faço questão em ser o primeiro a } \\
\text { comprar algo que seja lançamento }\end{array}$ \\
\hline 02 & $\begin{array}{l}\text { Dos meus amigos sou o primeiro a procurar e } \\
\text { comprar um produto, pois isso me faz bem. }\end{array}$ \\
\hline 03 & $\begin{array}{l}\text { Acompanho intensamente as tendências do } \\
\text { mercado, pois gosto de produtos de valor }\end{array}$ \\
\hline 04 & $\begin{array}{l}\text { Fico frustrado quando não participo de uma } \\
\text { tendência, pois perco produtos de valores }\end{array}$ \\
\hline
\end{tabular}

Quadro 1: Variáveis utilizadas no estudo Fonte: Adaptado pelos autores conforme escalas citadas.

O presente estudo se caracterizou como um experimento de laboratório em que foi manipulado a promoção de vendas (com ou sem) e o fator, tipo de promoção (monetária - desconto, e; não monetária concurso de prêmios). Para avaliação do fator "promoção de vendas" optou-se pelo método whitin-subjects, enquanto para o fator "tipo de promoção" aplicou-se o método between-subjects.

Os participantes participaram aleatoriamente de cada um dos dois cenários possíveis, sendo que, primeiramente, era entregue um anúncio do netbook. Esse anúncio fazia uma breve descrição do produto, com seu valor divulgado e trazia cinco perguntas para avaliar a intenção de compra dos respondentes. Nessa situação não havia a manipulação de qualquer tipo de promoção.

Uma vez preenchido, o questionário era recolhido e o participante recebia novamente o anúncio do mesmo produto, com as mesmas características, porém, 
agora, com o destaque do percentual da promoção de desconto e o novo valor do produto (no caso da promoção monetária). Para o caso do cenário contendo uma promoção não monetária, era anunciado o mesmo produto, contendo as mesmas características e valor. No entanto, neste caso, com a chamada para um concurso de prêmio, no qual era divulgada a possibilidade do consumidor, ao comprar o produto, estar concorrendo a dez automóveis $0 \mathrm{~km}$. Além dessa informação, os respondentes eram submetidos às escalas de intenção de compra, percepção de marca fictícia e característica conspícua do consumidor evocado pelo produto em promoção (conforme já destacado).

\subsection{Controle das Variáveis Estranhas}

As variáveis estranhas constituem-se em um problema para o pesquisador, pois são variáveis que não afetam a independente, mas que podem interferir nos efeitos das dependentes. Em vista disso, alguns cuidados foram tomados para evitar os efeitos das variáveis estranhas neste estudo. Primeiramente, o produto ofertado continha uma marca fictícia, denominada de Alpha. Essa decisão tem como objetivo evitar que a influência de uma marca conhecida pudesse interferir na intenção de compra do consumidor e demais elementos estudados, haja vista que outros estudos já encontraram esta relação (KELLER, 1993; AAKER, 1998; KELLER; LEHMANN, 2006).

O acompanhamento pessoal do pesquisador na aplicação das pesquisas auxiliou no controle da interação entre os respondentes, haja vista que esta, de alguma forma, poderia ser uma variável a vir a interferir nos resultados da pesquisa. Outra questão importante controlada em razão da artificialidade do experimento foi o recurso financeiro disponível ao pesquisado, uma vez que esta variável é um elemento influenciador sobre o aumento de incidência de compra (IYER, 1989; HAUSMAN, 2000).

Controlaram-se, também, as possíveis influências sociodemográficas nos resultados da pesquisa, por exemplo, renda, sexo e idade, já que a aplicação de estudos junto a estudantes permite obter certa homogeneidade da amostra e aumento da validade interna do experimento (PETERSON, 2001).

Por fim, foi identificado se as manipulações do experimento foram percebidas pelos participantes. Isso porque, a percepção da manipulação é um pressuposto para testar relações de causa e efeito. Para isso, a última pergunta do instrumento, questionava qual o era o percentual de desconto ofertado na situação hipotética (no caso do cenário de promoção monetária) e quantos automóveis o concurso de prêmios sorteava (no caso do cenário de promoção não monetária). De posse dessas informações, foram excluídos da amostra final, aqueles participantes que não perceberam as referidas manipulações, que eram: $37 \%$ de desconto (promoção monetária) e dez automóveis (promoção não monetária).

\section{Análise dos Resultados}

Tendo como objetivo avaliar a manipulação efetivada neste estudo, foram identificados os consumidores que não perceberam os efeitos trabalhados nos cenários estudados, que eram: (i) promoção monetária: $37 \%$ de desconto; (ii) promoção não monetária: sorteio de dez automóveis $0 \mathrm{Km}$. Nesta etapa, foram identificados e conseqüentemente excluídos, 75 questionários que não observaram os efeitos manipulados, pois informaram percentuais de desconto ou número de automóveis sorteados, diferente, dos dados constantes nos questionários.

Em seguida e ainda objetivando preparar os dados para as análises subsequentes, efetivou-se a purificação da base de dados, excluindo-se questionários de respondentes considerados outliers. Para esse caso, foram excluídos questionários com índice alto de não preenchimento (39), outliers univariados (35) e multivariados (36).

Com base no universo total dos questionários válidos - 303 elementos - efetivou-se a análise estatística descritiva para compreender as características amostrais da pesquisa. Sinteticamente, obteve-se uma amostra com uma maior concentração de renda mensal individual entre $R \$ 1.000$ a $R \$ 3.000$ (49,5\%), com idade entre 26 a 30 anos (44,9\%), do sexo feminino $(52,5 \%)$.

\subsection{Teste e Discussão da H1 e H1a: promoções monetárias versus não monetárias}

As primeiras hipóteses previam que as técnicas de promoção de vendas iriam influenciar a decisão de compra do consumidor, e ainda, que a técnica de promoção de desconto (monetária) influenciaria 
com mais força a compra, em relação a promoção de concurso de prêmios (não monetária), de acordo com alguns estudos (GILBERT; JACKARIA, 2002; ALVAREZ; CASIELLES, 2005).

A partir disso, efetivou-se a análise de test $t$ para avaliar a influência da promoção, independentemente do tipo, na intenção de compra do consumidor e, em seguida, a análise de variância (ANOVA) para testar a hipótese que previa uma diferença maior e, estatisticamente significante, para o grupo exposto a promoção monetária (desconto). A Figura 1 apresenta os resultados obtidos.

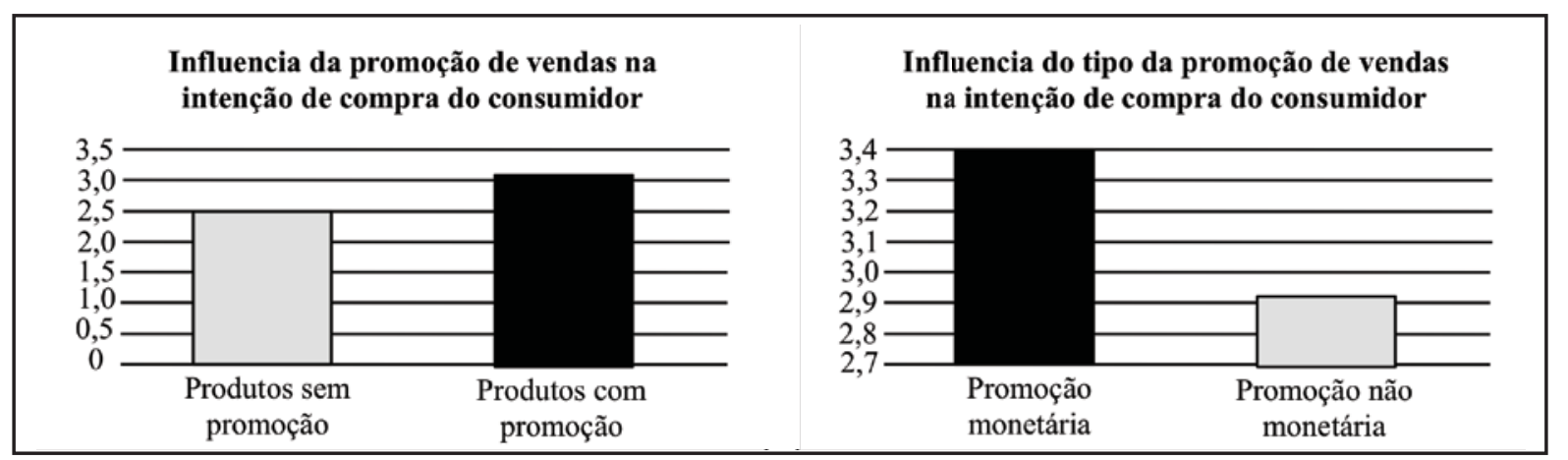

Figura 1: Teste das hipóteses $\mathrm{H} 1$ e $\mathrm{H} 1 \mathrm{a}$

Fonte: Elaborado pelos autores deste artigo com base nos resultados da pesquisa

Diante da Figura 2, observa-se, primeiramente, que a promoção de vendas, independente do seu tipo (monetária ou não monetária), influenciou na intenção de compra do consumidor (média produto sem promoção $=2,47$; desvio padrão produto sem promoção $=0,72 ;$ média produto com promoção $=$ 3,15 ; desvio padrão produto com promoção $=0,73$; $t=-10,397 ; \mathrm{p}<0,00)$. Tal resultado traz suporte para confirmação da Hipótese H1.

Em seguida, observa-se que a promoção monetária de desconto teve maior força na influência da intenção de compra do consumidor do que a promoção de vendas não monetária (média promoção monetária $=3,41$; desvio padrão promoção monetária $=0,10$; Média promoção não monetária $=2,98$; desvio padrão promoção monetária $=0,09 ; f=8,336$; $\mathrm{p}$ $<0,00)$, ratificando a proposição da hipótese H1a.

Em termos acadêmicos, esse resultado vem ao encontro de conclusões de pesquisas anteriores, que demonstram o efeito positivo da promoção de vendas no comportamento do consumidor (GUPTA, 1988; ALVAREZ; CASIELLES, 2005; SANTINI et al., 2011; BOSCHETTI, 2012), assim como, reforçam as suposições teóricas de que a promoção de vendas é um elemento importante para influenciar o comportamento de compra de um cliente (BLATTBERG; NESLIN, 1990), estimular demanda de consumo e encorajamento de troca de marca (GUPTA, 1988; DAVIS et al., 1992). Além disto, ficou reforçada a suposição da maior preferência e força na influencia da intenção de compra do consumidor, para as promoções de caráter monetário (GILBERT; JACKARIA, 2002; ALVAREZ; CASIELLES, 2005).

Em termos gerenciais, os resultados vêm ratificar elementos importantes no gerenciamento de estoques, por demonstrar que a promoção de vendas e, especialmente, a monetária, pode ser um elemento chave para o estímulo de vendas de produtos estocados e, ainda, uma ferramenta de diferenciação em categorias de alta competitividade de produtos (JONES, 2008). Além disso, gestores podem avaliar o uso desta ferramenta promocional para estimular a experimentação de novos produtos (NBUDISI; MOI, 2005), a elevação do nível de compra dos bens promocionados (COTTON; $\mathrm{BABB}, 1978)$ e o aumento do volume de vendas em curto prazo (BLATTBERG; NESLIN, 1990).

\subsection{Teste e Discussão da H2 e H2a: a moderação na percepção da marca}

Antes de efetivar o teste das hipóteses $\mathrm{H} 2, \mathrm{H} 2 \mathrm{a}$, H3 e H3a, realizou-se testes preliminares para verificar a adequação do modelo proposto, dentro do escopo de análise citada. Nesse sentido, foi possível observar índices adequados de curtose e assimetria, além da validade convergente e discriminante dos construtos. $\mathrm{Da}$ mesma forma, detectou-se a adequação dos índices das cargas fatoriais das variáveis manifestas, assim como 
para os índices de confiabilidade simples e composta dos fatores utilizados em análise.

Em relação aos parâmetros de ajustamento do modelo integrado, estes também foram satisfatórios (ARCABUCKE, 2008) - Qui-quadrado = 212,713; graus de liberdade $=75$; nível de probabilidade $=$ 0,000; qui-quadrado / graus de liberdade $=2,83 ; \mathrm{CFI}$ $=0,969 ; \mathrm{NFI}=0,953 ; \mathrm{RMSEA}=0,078$. Além disso, os resultados apontam relações significativas para o desvio padrão e teste $Z$ do modelo integrado.

Quanto à análise das hipóteses que previam efeitos moderadores, utilizou-se a análise de modelagem de equações estruturais multigrupos. Nessa perspectiva é possível estabelecer a categorização de uma variável (nominal ou ordinal) em dois ou mais grupos, que são classificados como hipótese moderadora (KRÜLL; MacKINNON, 2001). No caso desta pesquisa, a hipótese moderadora propõe diferença na intenção das trajetórias (paths) entre os construtos estabelecidos por conta da intensidade referente ao tipo da promoção de vendas. A manipulação do tipo de promoção (monetária e não monetária) se deu a partir da aplicação do experimento, em que um grupo foi exposto a uma promoção de desconto (monetária) e outro, a uma promoção não monetária (concurso de prêmios).
Realizada a separação dos grupos, e com o objetivo de testar o efeito moderador deste comportamento no modelo proposto (tipo de promoção), estimaram-se os parâmetros simultaneamente para cada grupo.

Na hipótese H2, era prevista uma relação positiva entre a promoção de vendas e a percepção de marca. Os resultados $(\beta=0,229 ; p<0,001)$, confirmam a referida suposição. Em seguida a Figura 2 , exibe os resultados encontrados em relação ao teste da hipótese H2a, em que era previsto um efeito moderador do tipo de promoção de vendas (monetária e não monetária) na relação entre percepção de marca e intenção de compra do produto em promoção.

Com esse intuito, foi seguido o procedimento de Aiken e West (1991), no qual utiliza-se o coeficiente não padronizado em uma plotagem para visualização da interação - neste caso, o tipo de promoção. $\mathrm{O}$ teste envolve dividir o moderador em um grupo alto (dois desvios padrões maior que a média) e um grupo reduzido (dois desvios padrões a menos que a média). Portanto, o slope e o intercepto da regressão da percepção da marca sobre intenção de compra dependem dos valores assumidos pelo moderador "tipo de promoção" (WEST et al., 1996).

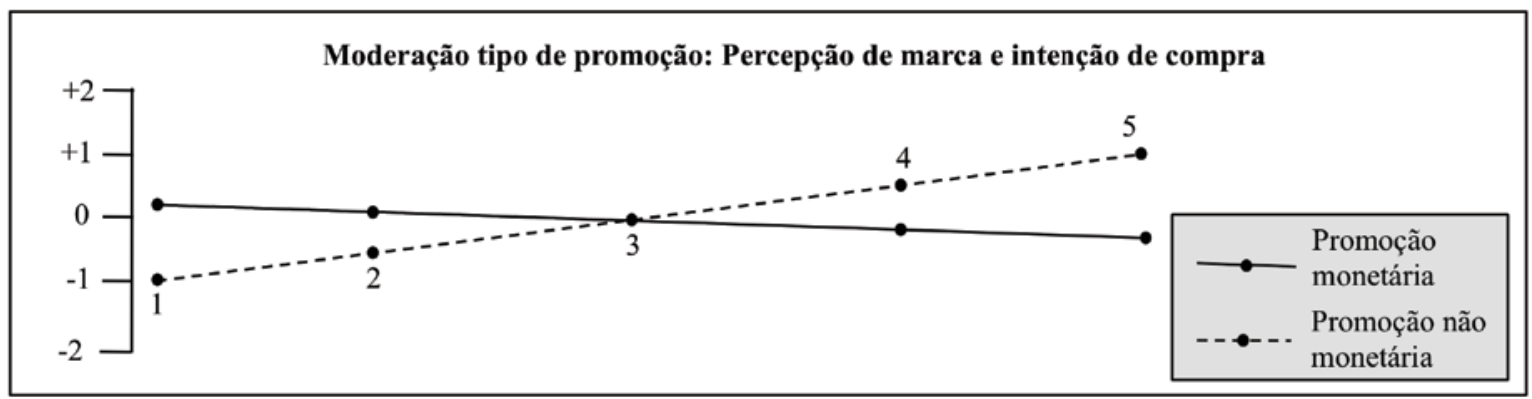

Figura 2: Moderação do tipo de promoção na relação entre percepção da marca e a intenção de compra do produto em promoção (H2a)

Fonte: Elaborado pelos autores deste artigo com base nos resultados da pesquisa

Visualiza-se, por meio da Figura 2, que a inclinação é mais alta, para a promoção não monetária $(\beta=0,436 ; p<0,001)$ do que para a promoção monetária ( $\left.\beta=-0,049^{\mathrm{ns}}\right)$, ou seja, observa-se o efeito esperado. Salienta-se que o índice de $\mathrm{X}^{2}$ referente à diferença estatística dos modelos foi significativo ( $p<0,001$ ), o que corrobora a suposição da hipótese $\mathrm{H} 2 \mathrm{a}$.

Os resultados ratificam pesquisas anteriores, por exemplo, Pauwels et al. (2002) e Delvecchio et al.
(2006), em que são pautadas a relação positiva entre as ações de promoção de vendas e marca, além de consolidar os efeitos mais positivos sobre a marca, para as campanhas de caráter não monetário (DARK; CHUNG, 2005; BOSCHETTI, 2012).

Em termos gerenciais, os achados são importantes, uma vez que não se torna recomendável a utilização, em demasia, de campanhas monetárias, como é o exemplo de desconto, em marcas tradicionalmente reconhecidas, pois isto poderá acarretar, em longo 
prazo, numa depreciação da imagem, considerando que os consumidores podem se tornar sensíveis ao preço (LATTIN; BUCKLIN, 1989; EHERENBER et al., 1994). Por outro lado, as campanhas não monetárias, possivelmente, não trarão estes efeitos negativos, considerando que estas ações agregarão valor original ao produto, sem manipular o preço tradicional (SANTINI, 2008; BOSCHETTI, 2012).

\subsection{Teste e Discussão da H3 e H3a: a moderação no consumo conspícuo}

A hipótese $\mathbf{H 3}$ previa uma relação positiva entre a evocação conspícua e a intenção de compra de um produto em promoção de vendas, fato este, confirmado $(\beta=0,249 ; p<0,001)$. Na Figura 3, utilizando a mesma metodologia detalhada anteriormente, apresenta-se o teste da hipótese H3a, para avaliar a influência moderadora do tipo de promoção de vendas na relação entre evocação conspícua e intenção de compra do produto. Para essa situação, supõe-se que a referida relação seja mais forte para a promoção de vendas não monetária.

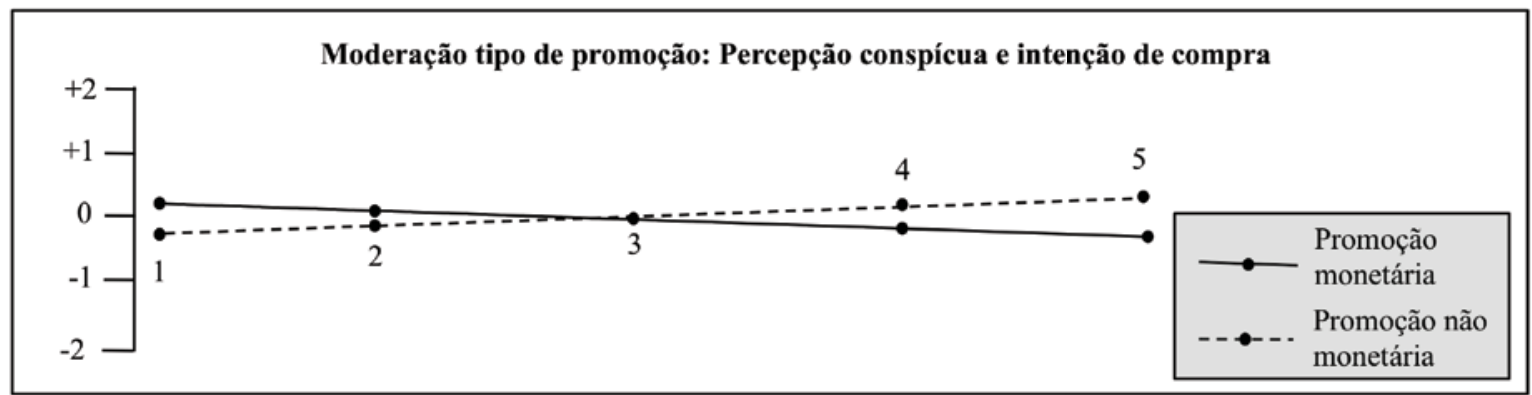

Figura 3: Moderação do tipo de promoção na relação entre evocação conspícua e intenção de compra do produto em promoção (H3a)

Fonte: Elaborado pelos autores com base nos resultados da pesquisa

A Figura 3 demonstra que a inclinação é mais alta para a promoção de vendas do tipo não monetária ( $\beta$ $=0,241 ; p<0,001)$ do que monetária $\left(\beta=-042^{\text {ns }}\right)$, ou seja, os índices dão sustentação para a hipótese $\mathrm{H} 3 \mathrm{a}$, uma vez que houve diferença significativa entre os modelos $\left(X^{2}<0,028\right)$.

Os achados consolidam a suposição de Chandon et al. (2000) e Kwok e Uncles (2005) de que os benefícios hedônicos são mais sutis (intrínsecos), ou seja, se vinculam aos aspectos emocionais e experienciais; sendo que os bens hedônicos são apreciados sem levar em conta os aspectos práticos, fato este bastante vinculado ao comportamento conspícuo (SCOTT et al., 2013). Considerando que a promoção monetária está bastante vinculada a preço e, por consequência, a um benefício visível e prático do produto, esta relação está mais atrelada aos aspectos utilitários do que aos hedônicos, este último, melhor relacionado com a promoção não monetária (concurso de prêmios).

Ademais, pode-se sugerir que um alto desconto (promoção monetária), poderá descredenciar uma das características principais do consumo conspícuo, que é a exclusividade associada ao bem (HIRSCHMAN; HOLBROOK, 1982; SPANGENBERG et al., 1997), podendo ser, ainda, um obstáculo significativo no caminho da formação das necessidades de prazer e excitações, essenciais na formação do consumo hedonista (DHAR; e WERTENBROCH, 2000; RAMANATHAN; MENON, 2006). Por fim, os resultados apresentados nesta pesquisa, permitem sugerir que a promoção monetária enfraquece a evocação do consumo conspícuo destacados por Arnold e Reynolds (2003), como o sentimento de pertencimento a certos grupos e a adesão para com estilos inovadores. A razão disso é que o fato de existir um abatimento no preço original de um produto poderá facilitar a adesão ao bem, ampliando esta possibilidade para os mais diversos grupos e classes sociais.

No âmbito gerencial, torna-se interessante pensar que, para produtos ou serviços de percepções mais hedônicas devem ser evitadas as promoções monetárias e estimuladas as ações não monetárias. 
Os resultados sugerem ainda, que as promoções de vendas e especialmente as de natureza não monetária, podem provocar uma maior excitação para o consumo conspícuo (HIRSCHMAN; HOLBROOK, 1982). Outra suposição interessante está vinculada à utilização desta ferramenta para lançamento de novos produtos, haja vista que este sentimento está relacionado à percepção hedônica assim como para o sentimento conspícuo (VENKATRAMAN; PRICE, 1990; KIM, 2008) e os resultados demonstraram relações positivas desta percepção com a intenção de compra do produto em promoção de vendas.

\section{Considerações Finais}

O presente trabalho objetivou analisar o efeito moderador do tipo de promoção (monetária ou não monetária) na intenção de compra. Especificamente, foram avaliados os efeitos na percepção de marca e evocação conspícua neste processo. Em termos acadêmicos, o estudo contribui para preencher lacunas identificadas na literatura, por exemplo, a investigação de fatores moderadores que podem potencializar ou minimizar o impacto da promoção de vendas no comportamento do consumidor (LOW; MOHR, 2000; FREO, 2004; ALVAREZ; CASILLES, 2005), a investigação de ações de marketing que podem se relacionar positivamente com a percepção de marca (LAROCHE et al., 2003) e de consumo conspícuo (CHAUDHURI et al., 2011).

Quanto aos aspectos gerenciais, o estudo procura contribuir com novos subsídios aos gestores, para o planejamento e execução de suas campanhas promocionais, uma vez que muitas decisões costumam ser realizadas com base em intuições dos gerentes em detrimento aos aspectos científicos (D'ASTOUS; LANDREVILLE, 2003). A necessidade de fundamentar com estudos empíricos estas decisões deve ser observada com um cuidado cada vez maior, considerando que os investimentos realizados em ações de promoção de vendas vêm sendo anualmente incrementados (TEUNTER, 2010; WIERENGA; SHOETHOUDT, 2010) e que as técnicas de promoções de vendas vêm recebendo, por parte de muitas empresas, mais investimentos do que a publicidade ou eventos (LOW; MOHR, 2000; LUK; YIP, 2008). Isso, porque, com frequência, as empresas estão inseridas num mercado competitivo $e$ as ações promocionais servem como norteador de uma diferenciação do concorrente e um estimulador de consumo (GUPTA, 1988; BLATTBERG; NESLIN, 1990; GILBERT; JACKARIA, 2002).

O tema "promoção de vendas" e sua investigação ainda possuem um amplo campo para debates, pois, apesar de ser uma técnica bastante utilizada e considerada no campo gerencial, é um assunto pouco analisado no âmbito acadêmico ( $D^{\prime}$ ASTOUS; LANDREVILLE, 2003; ALVAREZ; CASIELLES, 2005). Com esse objetivo, o estudo aqui proposto procurou contribuir para um melhor entendimento a respeito de comportamentos relacionados à promoção de vendas e sua relação com a intenção de compra do consumidor.

O estudo encontra limitações metodológicas, detectadas ao longo do trabalho. As primeiras limitações que podem ser destacadas dizem respeito à técnica de amostragem utilizada, ao público ao qual a pesquisa foi aplicada e, ainda, à característica do experimento utilizado. A composição da amostra por estudantes de um único curso e faculdade, apesar de ter sido justificada, pode causar algum viés nos resultados. Pesquisas posteriores poderão utilizar amostras com características diferentes, para ratificar ou não os resultados encontrados nesta pesquisa. Interessante, também, seria pensar na possibilidade de empresas aplicarem pesquisas como esta aos seus clientes. A técnica de amostragem utilizada, não probabilística, também não permite atribuir os resultados de forma generalizada dentro da população investigada. Sendo assim, pode-se sugerir a aplicação de pesquisas com técnicas de amostragem probabilística, permitindo generalização para a amostra utilizada. Quanto à característica do experimento utilizado, mesmo com as justificativas apresentadas no capítulo de metodologia, admite-se que ele tem limitações para confirmar a validade externa da pesquisa. Por essa razão, é de bom senso sugerir e realizar pesquisas que contemplem experimentos de campo para ratificar ou não os resultados aqui detectados.

Apesar das limitações destacadas, este trabalho contribui para um melhor e maior entendimento das influências exercidas pelas promoções de vendas no comportamento do consumidor, fomentando novas discussões teóricas a respeito do assunto, assim como, contribuir com os gestores de empresas para um melhor planejamento. 


\section{REFERÊNCIAS}

AAKER, D. A. Measuring brand equity across products and markets. Californian Management Review, [S.I.], v. 38, n. 3, p. 102-120, 1996.

AIKEN, W.; WEST, B. Multiple Regression: testing and interpreting interactions. Newbury Park, CA: Sage, 1999.

ALVAREZ, B.; CASIELLES, R. V. Consumer Evaluations of Sales Promotion: the Effect on Brand Choice.

European Journal of Marketing, [S.I.], v. 39, n. 1/2, p. 54-73, 2005.

ARBUCKLE, J. L. Amos 17 user's guide. Chicago, IL, SPSS, 2008.

BAWA, K.; SHOEMAKER, R. W. The Effects of a Direct Mail Coupon on Brand Choice Behavior. Journal of Marketing Research, [S.I.], v. 24, 1987.

\section{BLATTBERG, R. C.; NESLIN, S. Sales Promotion} Concepts, Methods, and Strategies. New Jersey: Prentice-Hall, 1990.

BLATTBERG, R. C.; WISNIEWSKI, K. J. Price-induced Patterns of Competition. Marketing Science, [S.I.], v. 8, n 4, p. 81-100, 1989.

BOSCHETTI, R. B. Promoção de Vendas não monetária e seus efeitos na intenção de compra e na escolho da marca de serviços financeiros. 2012. p.1 - 139. Dissertação (Mestrado) - Pontifícia Universidade Católica do Rio Grande do Sul, Rio Grande do Sul.

\section{BRUNER, G. C. II; HENSEL, P. J. Marketing Scales}

Handbook: a Compilation of Multi-items Measures. Chicago: American Marketing Association, 2, 1998.

\section{BUIL, I.; CHERNATONY, L.; MONTANER, T. Factors} influencing consumer evaluations of gift promotions. European Journal of Marketing, [S.l.], v. 47, n. 3/4, p. 574-595, 2013.

CAMPBELL, L.; DIAMOND, W. D. Framing and Sales Promotion: the Characteristics of a "Good Deal". Journal of Consumer Marketing, [S.l.], p. 25-31, 1990.

CHANDON, P.; WANSINK, B.; LAURENT, G. A Benefit Congruency Framework of Sales Promotion Effectiveness. Journal of Marketing, [S.I.], v. 64, n. 4, p. 65-78, 2000.
CHUNG, E.; FISCHER, E. When conspicuous consumption become inconspicuous: the case of the migrant Hong Kong consumers. Journal of Consumer Marketing, [S.l.], v. 18, n. 6, p. 474-487, 2001.

COTTON, B. C.; BABB, E. M. Consumer Response to Promotion Deals. Journal of Marketing, [S.l.], v. 42, p. 109-113, 1978.

D’ASTOUS, A.; LANDREVILLE, L. An Experimental Investigation of Factors Affecting Consumers' Perceptions of Sales Promotions. European Journal of Marketing, [S.I.], v. 37, n. 11/12, 2003.

DARKE, P. R.; CHUNG, C. M. Y. Effects of Pricing and Promotion on Consumer Perceptions: it Depends on how You Frame it. Journal of Retailing, [S.I.], v. 81, n. 1, p. 35-47, 2005.

DAVIS, S.; INMAN, J. J.; McALISTER, L. Promotion has a Negative Effect on Brand Evaluations-or does it? Additional Disconfirming Evidence. Journal of Marketing Research, [S.l.], v. 24, p. 143-148, 1992.

DELVECCHIO, D.; HENARD, D. H.; FRELING, T. H. The Effect of Sales Promotion on Post-promotion Brand Preference: a meta-analysis. Journal of Retailing, [S.I.], v. 82, n. 3, p. 203-213, 2006.

DIELS, J. L.; WIEBACH, N.; HILDEBRANDT, L. The impact of promotions on consumer choices and preferences in out-of-stock situations. Journal of Retailing and Consumer Services, [S.l.], v. 20, p. 587-598, 2013.

EHERENBERG, A. S. C; HAMMOND, K. A.; GOODHARDT, G. J. The After-effect of Price Related Consumer Promotions. Journal of Advertising Research, [S.l.], v. 34, n. 4, p. 11-21, 1994.

ERDEN, T.; SUN, B. An empirical investigation of the spillover effects of advertising and sales promotions in umbrella branding. Journal of Marketing Research, [S.l.], v. 39, n 4, 2002.

ESTEBAN-BRAVO, M.; MUGICA, J. M.; VIDAL-SANZ, J. Magazine Sales Promotion. Journal of Advertising, [S.l.], v. 38, n. 1, p. 137-146, 2009.

FISCHER, E.; ARNOLD, S. J. More than a Labor of Love: Gender Roles and Christmas Shopping. Journal of Consumer Research, [S.l.], v. 17, p. 333-345, 1990. 
FORSYTHE, S. M. Effect of private, designer, and national brand manes on shoppers'perception of apparel quality and price. Clothing and Textiles Research Journal, [S.l.], v. 9, p. 1-6, 1991.

FREO, M. The impact of sales promotions on store performance: a structural vector autoregressive approach. Statistical Methods \& Applications, [S.I.], v. 14, n. 2, 2005.

GARRETSON, J. A.; BURTON, S.; CLOW, K. E. The Influence of Coupon Face Value on Service Quality Expectations, Risk Perceptions and Purchase Intentions in the Dental Industry. The Journal of Services Marketing, [S.l.], v. 13, (1), p. 59-72, 1999.

GEDENK, K.; NESLIN, S. A. The Role of Retail Promotion in Determining Future Brand Loyalty: Its Effect on Purchase Event Feedback. Journal of Retailing, [S.l.], v. 75, n. 4, p. 433-459, 1999.

GILBERT, D. C.; JACKARIA, N. The Efficacy of Sales Promotions in UK Supermarkets: a Consumer View. International Journal of Retail \& Distribution Management, [S.l.], v. 30, n. 6/7, 2002.

GUPTA, S. Impact of Sales Promotions on When, What, and How Much to Buy. Journal of Marketing Research, [S.l.], v. 25, p. 342-355, 1988.

HALVENA, W. J.; HOLBROOK, M. B. The Varieties of Consumption Experience: Comparing Two Typologies of Emotion in Consumer Behavior. Journal of Consumer Research, [S.l.], p. 394-404, 1986.

HAUSMAN, A. A Multi-method Investigation of Consumer Motivations in Impulse Buying Behavior. Journal of Consumer Marketing, [S.l.], v. 17, p. 403-419, 2000.

HIRSCHMAN, E. C.; HOLBROOK, M. B. Hedonic Consumption: Emerging Concepts, Methods and Propositions. Journal of Marketing, [S.l.], v. 46, p. 92107, 1982.

HOFFMAN, D. L.; NOVAK, T. P. Marketing in Hypermedia Computer-mediated Environments: Conceptual Foundations. Journal of Marketing, [S.l.], v. 60, p. 5068, 1996.
HORVÁTH, C.; FOK, D. Moderating Factors of Immediate, Gross, and Net Cross-Brand Effects of Price Promotions. Marketing Science, [S.l.], v. 32, n. 1, p. 127-152, 2013.

IYER, E. Unplanned Purchasing: Knowledge of Shopping Environment and Time Pressure. Journal of Retailing, [S.l.], v. 65, p. 40-57, 1989.

KANG, J.; PARK-POAPS, H. Hedonic and Utilitarian Shopping Motivations of Fashion Leadership. Journal of Fashion Marketing and Management, [S.I.], v. 14, n. 2, p. 312-328, 2010.

KELLER, K. L. Conceptualizing, measuring, and managing customer-based brand equity. Journal of Marketing, [S.I.], v. 57, p. 1-22, 1993.

KELLER, K. L.; LEHMANN, D. R. Brands and Branding: Research Findings and Future Priorities. Marketing Science, [S.l.], v. 1, n. 6, p. 740-759, 2006.

KIM, Y. H. An Empirical Examination of Consumers Innovation Adoption: The Role of Innovativeness, Fashion Orientation, and Utilitarian and Hedonic Consumers Attitudes. 2008. Dissertação (Mestrado em Negócios) - University of North Carolina, 2008.

KRÜLL, J. L.; McKINNON, D. P. Multilevel modeling of individual and group level mediated effects. Multivariate Behavioral Research, [S.I.], v. 36, n. 2, p. 249-277, 2001.

KWOK, S.; UNCLES, M. Sales Promotion Effectiveness: the Impact of Consumer Differences at an Ethnicgroup Level. The Journal of Product and Brand Management, [S.l.], v. 14, n. 2/3, 2005.

LATTIN, J. M.; BUCKLIN, R. E. Reference Effects of Price and Promotion on Brand Choice Behavior. Journal of Marketing Research, [S.I.], v. 26, p. 299-310, 1989.

LARAN, J.; TSOIRUS, M. An Investigation of the Effectiveness of Uncertainty in Marketing Promotions Involving Free Gifts. Journal of Marketing, [S.I.], v. 77, p. 112-123, 2013.

LAROCHE, M. et al.A Model of Consumer Response to Two Retail Sales Promotion Techniques. Journal of Business Research, [S.l.], v. 56, p. 513-522, 2003. 
LEE, C. W. Sales Promotions as Strategic Communication: the Case of Singapore. The Journal of Product and

Brand Management, [S.I.], v. 11, n. 2/3, 2002.

LIAO, S. The Effects of Nonmonetary Sales Promotions on Consumer Preferences: The Contingent Role of Product Category. Journal of American Academy of Business, Cambridge, v. 8, n. 2, 2006.

LI, X. How Brand Kwowledge influence consumers' purchase intentions. 2004. Tese (Doutorado) - Alburn University, 2004.

LOW, G. S.; MOHR, J. J. Advertising us Sales Promotion: a Brand Management Perspective. Journal of Product and Brand Management, [S.I.], v. 9, n. 6, 2000.

LOWE, B.; BARNES, B. R. Consumer perceptions of monetary and non-monetary introductory promotions for new products. Journal of Marketing Management, [S.l.], v. 28, n. 5-6, p. 629-651, 2012.

LUK, S.; YIP, L. The Moderator Effect of Monetary Sales Promotion on the Relationship Between Brand Trust and Purchase Behaviour. Journal of Brand Management, [S.l.], v. 15, n. 6, 2008.

MACHLEIT, K. A.; WILSON, R. D. Emotional feelings and attitude toward the advertisement: the roles of brand familiarity and repetition. Journal of Advertising, [S.I.], v. 17, n. 3, p. 27-35, 1988.

NBUDISI, N. O.; MOI, C. T. Customers Behavioural Responses to Sales Promotion: the Role of Fear of Losing Face. Asia Pacific Journal of Marketing and Logistics, [S.l.], v. 17, n. 1, 2005.

NESLIN, S. A.; SHOEMAKER, R. W. An Alternative Explanation for Lower Repeat Rates After Promotion Purchases. Journal of Marketing Research, [S.I.], v. 26, p. 205-213, 1989.

NIJS, V. R. et al. The Category demand Effects of Price Promotions. Marketing Science, [S.I.], v. 20, n. 1, p. 1-22, 2001.

PETERSON, R. On the Use of College Students in Social Science and Research: Insights from a Second-order meta-analysis. Journal of Consumer Research, [S.I.], v. 28, 2001.
PAUWELS, K. et al. New Products, Sales Promotions, and Firm Value: the Case of the Automobile Industry. Journal of Marketing, [S.l.], v. 68, n. 4, p. 142-156, 2004.

PATSIAOURAS, G.; FITCHETT, J. A. The evolution of conspicuous consumption. Journal of Historical Research in Marketing, [S.l.], v. 4, n. 1, p. 154-176, 2012.

RAJAGOPAL, M. Point-of-sales promotions and buying stimulation in retail stores. Journal of Database

Marketing \& Customer Strategy Management, [S.l.], v. 15, n. 4, 2008.

OMOTAYO, O. Sales Promotion and Consumer Loyalty: A Study of Nigerian Tecommunication Industry. Journal of Competitiveness, [S.l.], v. 4, p. 66-77, 2011.

SANTINI, F. O. Uma análise da influencia da promoção de vendas de desconto na intenção de compra do consumidor e os efeitos moderadores da atratividade. 2013. p. 1 - 199. Tese (Doutorado) Pontifícia Universidade Católica do Rio Grande do Sul, Rio Grande do Sul, 2013.

SANTINI, F. O.; SAMPAIO, C. H.; PERIN, M. G. Promoção de Desconto: seus efeitos na compra por impulso e nas intenções de recompra. In: CONGRESSO LATINO-AMERICANO DE VAREJO - CLAV, 4, São Paulo, Anais... São Paulo, 2011. p. 1-16.

SANTINI, F. O. et al. Percepção de valor, atratividade e intenção de compra: revisitando as técnicas de promoção de vendas. Revista Brasileira de Gestão de Negócios, [S.l.], v. 17, n. 57, p. 1.173-1.192, 2015.

SANTINI, F. O. et al. Moderating effects of sales promotion types. Brazilian Administration Review, [S.I.], v. 12, n. 2, p. 169-189, 2015.

SANTINI, F. O. et al. Uma análise da influência da promoção de vendas de desconto na intenção de compra do consumidor e os efeitos moderadores da atratividade.

Revista de Administração da USP, [S.l.], v. 50, n. 4, p. 416-431, 2015.

SCOTT, M. L.; MENDE, M.; BOLTON, L. E. Judging the book by its cover? How consumers decode conspicuous consumption cues in buyer-seller relationships. Journal of Marketing Research, [S.1.], v. p. 334-347, 2013. 
SHUKLA, P. Conspicuous consumption among middle age consumers: Psychological and brand antecedents.

Journal of Product \& Brand Management, [S.l.], v. 17, n., 1, p. 25-38, 2008.

TAN, S.; CHUA, S. H. While Stocks Last! Impact of Framing on Consumers Perception of Sales Promotions.

The Journal of Consumer Marketing, [S.l.], v. 21, n. 4/5, 2004.

TAYLOR, G. A.; NESLIN, S. A. The Current and Future Sales Impact of a Retail Frequency Reward Program. Journal of Retailing, [S.1.], v. 81, n. 4, p. 293-305, 2005.

TEUNTER, L. H. Analysis of sales promotion effects on household purchasing behavior. ERIM PhD Research Series in Management. Erasmus University Rotterdam, [S.l.], p. 262-272, 2002.

VENKATRAMAN, M. P.; PRICE, L. P. Differentiating between Cognitive and Sensory Innovativeness: Concepts, Measurement and their Implications. Journal of Business Research, [S.1.], v. 20, p. 293-315, 1990.

WEINBERG, P.; GOTTWALD, W. Impulsive Consumer Buying as a Result of Emotions. Journal of Business Research, [S.l.], v. 10, p. 43-57, 1982.

WEST, S. G.; AIKEN, L. S.; KRULL, J. L. Experimental Personality Designs: Analyzing Categorical by Continuous Variable Interactions. Journal of Personality, [S.1.], p. 1-48, 1996.

WIERENGA, B.; SOETHOUDT, H. Sales Promotions and Channel Coordination. Original Empirical Research, [S.l.], p. 383-397, 2010.

WILSON, T. D.; ARONSON, E.; CARLSMITH, K. The art of laboratory experiment. Handbook of Social

Pshychology, [S.I.], 2010.

WINER, R. A. Reference Price Model of Demand for Consumer Durables; Preliminary Developments.

Marketing Science, [S.I.], v. 4, p. 74-90, 1985.

WINKELMANN, R. Conspicuous consumption and satisfaction. Journal of Economic Psychology, [S.l.], v. 33, p. 183-191, 2012. 